\title{
A Compact Continuum Manipulator System with Enhanced Steering Abilities for Robot-Assisted Surgery*
}

\author{
Peng Qi, Chunsong Zhang, Jianmin Li, Zheng Li, Jian S. Dai, and Kaspar Althoefer
}

\begin{abstract}
Medical robotics plays an increasingly important role in minimally invasively surgery (MIS), where access is usually through a small incision on the body. This paper introduces the conceptual design of a compact MIS robot that integrates a continuum manipulator with remote center-of-motion (RCM) mechanism. This robotic system allows surgical instruments to enhance the dexterity inside the patient's body - a desirable feature in MIS and potentially improving the safety. We derive the integrated kinematics of the entire system, describing both positions and orientations of the end-effector in the global coordinate system. Simulations are conducted in MATLAB to analyze the reachable workspace of the entire system and its separate subsystems. The comparisons highlight the "dexterous operation area" of the proposed system and its ability of local orientation adjustment. Finally, an overview of preliminary tests with a 3D-printed prototype validating the design is given.
\end{abstract}

\section{INTRODUCTION}

In the last two decades robotics has contributed lots of new treatment options to conventional surgical procedures [1], [2]. Many task-orientated surgical robots have been developed and they effectively boosted the advancement of minimally invasive surgery (MIS) techniques. First, the MIS-specialized mechanism designs enable an increased maneuverability in the confined MIS environments; further, the robotic features enhance the precision and repeatability of MIS operations. Particularly, continuum manipulators as a type of newly emerged robotic design offer great potential in their applications for medical usage [3], [4]. They are also called flexible manipulators and inspired by elephant trunks, snakes and octopus tentacles which possess unique advantages in terms of flexibility, dexterity, safety, and reduced weight [5]. Recently, there is an increasing trend of rigid straight shafts of medical devices being evolved into steerable tips such as flexible endoscope and laparoscopic tools; besides, flexible robot manipulators developed for specific surgical applications also spring up, such as concentric tube robots [6] and multi-backbone snake-like robots [7]. The history of the continuum manipulator and the current stage of knowledge in this field have been presented in review paper [8].

*Research supported by the FRC Tier 1 grant under the WBS NO R-397-000-156-112.

P. Qi is with the Department of Biomedical Engineering, Faculty of Engineering, National University of Singapore, Singapore 117583. (corresponding author to provide e-mail: peng.qi@nus.edu.sg)

C. Zhang, J. Li, and J. S. Dai are with the School of Mechanical Engineering, Tianjin University, China.

$\mathrm{Z}$. Li is with the Chow Yuk Ho Technology Centre for Innovative Medicine, Chinese University of Hong Kong, Shatin, Hong Kong.

J. S. Dai is also with the Centre for Robotics Research, Department of Informatics, King's College London, WC2R 2LS, United Kingdom.

$\mathrm{K}$. Althoefer is with the School of Engineering and Materials Science, Queen Mary University of London, Mile End Rd, E1 4NS, United Kingdom.
Robotic surgery is the application of engineering principles to surgical procedures. The goal of robotics here is not to take the robot to replace the surgeon, but to assist the surgeon in carrying out surgical procedures. The remote center-of-motion (RCM) mechanism is a general term of a class of MIS-specialized mechanisms [9]. It not only provides a pivoting 4-degree-of-freedom (DOF) motion centered at the small incision to fulfil functional requirements, but also can avoid the fulcrum point shifting due to control failure based on its own structural constraints. Therefore, RCM mechanisms have often been applied to single-port access (SPA) surgery.

There are many different types of mechanisms that can provide an RCM. The parallelogram-type mechanism is the most commonly used RCM mechanism [10]. The representative commercialized surgical robot $-d a$ Vinci system (Intuitive ${ }^{\circledR}$ ) -is embedded with this parallelogram-type RCM mechanism. Other RCM mechanism types (such as spherical linkage [11], parallel mechanism, gimbal, etc.) and the examples of their embodied MIS robots are explicitly summarized in [9]. It is concluded that in the phase of kinematic design of RCM mechanisms researchers mainly concern these aspects of safety, workspace volume, DOF, decoupled motion, etc. Usually an RCM mechanism acts as the laparoscope holder or attach other detachable grippers [12] - [14]. Its reachable workspace encompasses a cone-like 3D area and is always restricted to the RCM point.

In this paper, inspired by the design concept of macro-micro manipulator system [15], we propose a compact MIS robot that integrates a flexible continuum manipulator [16] with an RCM mechanism. It achieves both overall safe pivoting motion and local steering dexterity of surgical instruments. Furthermore, the continuum manipulator part and RCM mechanism part are fully actuated such that the kinematic redundancy leads the robot to reach the targeted position in more than one possible orientations. The entire system fulfils functional requirement of providing an RCM point and also enhances the manipulator's mobility especially for providing the ability of local orientation adjustment.

The continuum manipulator used in this system is based on the previous work [16]. Here, we decrease its diameter into 15 $\mathrm{mm}$ with an improved planar spring design, thus fitting a standard trocar port. The current prototype is 3D printed using acrylonitrile butadiene styrene (ABS) plastic material, but it limited the further miniaturization. Smaller size structures can be made of other elastic metallic materials, which will enable the applications towards minimally invasive operations through small portals. The novelty of this design is embodied in three curved legs suspending the central platform. It is this curved leg that has the potential to create a more compact planar spring design than the previous straight beam. More details of the continuum manipulator design will be seen in Section II. 


\section{CONCEPTUAL Design}

\section{A. Continuum Manipulator Design}

The continuum manipulator design utilizes the concept of linking multiple layers of planar springs [17] in series, which has been previously proposed in [16]. The bending of this type of continuum manipulator is based on the compliance of the planar spring. Each planar spring consists of a central platform, three curved legs, and a ring base [17]; they are indicated in Fig. 1. Two layers of planar springs construct a modular segment and this double-layer segmented design allows the length of the continuum manipulator to be easily changed. The curved legs are the flexible members, while other parts (central platform and outer frame) are theoretically rigid. Tendons (fishing line) through the tendon channel at the periphery are employed to actively actuate (bend or contract) the entire stack. The pulling force is exerted to the distal spring's platform, which will be transmitted to the proximal bottom support, and the load is distributed to each layer of planar spring generating local motions, thus the robot manipulator is articulated and bends continuously.

This new linked robot manipulator has demonstrated several advantages: it avoids all friction between the components of adjacent links; the construction is optimized to simplify control and allowing linear predictable bending even for large bends; the deformation utilizes the movability of the planar spring's central platform for each with two rotational DOFs and one extra DOF to allow the manipulator's contraction, effectively decoupling bending from contraction.

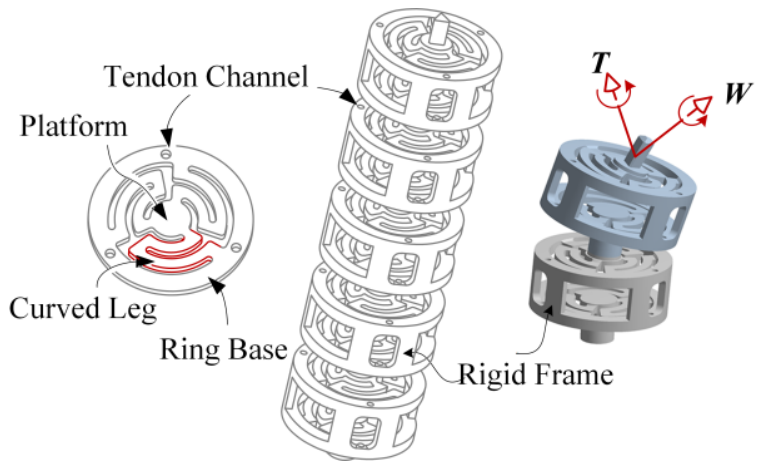

Figure 1. Schematics of the curved-leg planar spring (left), and continuum structure assembly (middle). The bending deformation of two double-layer modular segments is illustrated (right); a twist deformation $\boldsymbol{T}$ and a general wrench $\boldsymbol{W}$ on the central platform are located.

\section{B. Spherical Linkage RCM Design}

As introduced above, an RCM mechanism is used to perform pivoting 4-DOF motions based on the mechanical constraint; they are 3-DOF rotations centered at the minimally invasive incision point and 1-DOF translation passing through it into the patient's body. In many cases, spherical linkage mechanisms are commonly utilized to accomplish this desired motion [11], [18]. In spherical linkage mechanisms, all the linkages rotate about the center of the sphere and all the axes of joints intersect at the center of the sphere. Hence, this characteristic of spherical linkage mechanisms provides an ideally mechanical and physical constraint for the pivoting motion centered at the minimally invasive incision point. Furthermore, compare to other RCM mechanisms, such as parallel mechanisms and parallelograms [9], [10], in theory there is no need for a designer to add extra linkages into the spherical mechanism type of RCM mechanisms in order to maintain the pivoting motion. It is compact in terms of the criterion that the base frame is close to the incision point [12], [19]. Hence, this type of RCM mechanisms can provide a large operating space for the surgeons to avoid undesired collisions and less self-occupied space. Besides, it is simple-constructed and lightweight, thus easier to design and in some aspects reducing the control complexity in force feedback control system due to its small inertia.

Here, in the case for a general purpose surgical robot, a 3-bar linkage spherical mechanism (Link 1 acts as the rack of the mechanism in Fig. 2) is utilized to achieve the function of an RCM mechanism, where the pivoting point is exactly the sphere center. The conceptual design is illustrated in Fig. 2: two DOFs for rotary motions are actuated by motors; one DOF for the spinning motion and another one for translation currently are manual-adjusted but will be renovated with spinning and linear motors for next generation of design.

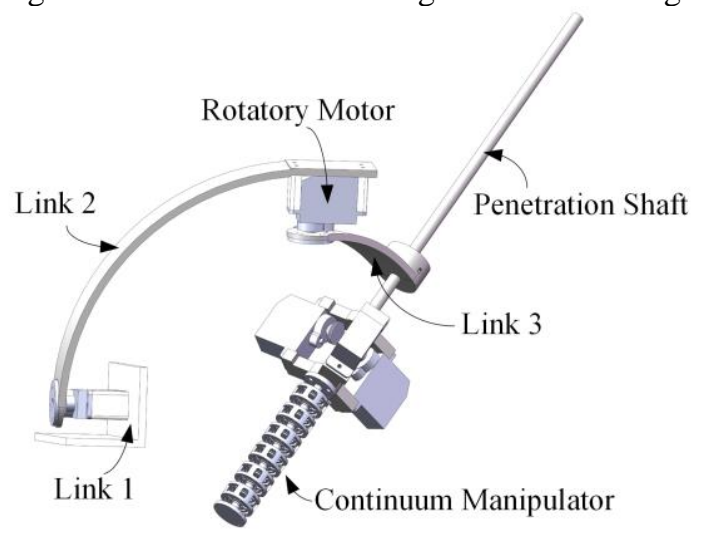

Figure 2. The conceptual design of a spherical linkage RCM mechanism with a continuum manipulator mounted for illustration.

\section{KINEMATICS}

\section{A. Coordinate Systems and Problem Formulation}

The RCM mechanism provides pivoting 4-DOF motions. As shown in Fig. 3, the global coordinate system $\boldsymbol{o}_{o}-\boldsymbol{x}_{o} \boldsymbol{y}_{o} \boldsymbol{z}_{o}$ is established in the center of this open-chain spherical linkage mechanism and the $\boldsymbol{y}_{o} \boldsymbol{z}_{o}$ plane coincides with the equatorial plane. And then for convenience, other local coordinate systems $\boldsymbol{o}_{i}-\boldsymbol{x}_{i} \boldsymbol{y}_{i} \boldsymbol{z}_{i}(i=1,2,3)$ are established on each actuated joints in sequence and all the origins $\boldsymbol{o}_{i}$ are placed at the coordinate system's origin - the center of the sphere. Axes $z_{i}$ are collinear with the axes of the joint $i$ and point to the outside of the spherical surface; axes $\boldsymbol{y}_{i}$ share the same direction with the cross product $\boldsymbol{z}_{i} \times \boldsymbol{z}_{i+1}$; axes $\boldsymbol{x}_{i}$ complete the right-handed coordinate systems, respectively. In particular, the axis $z_{o}$ is collinear with the axis $z_{1}$ and shares the same direction; the axis $y_{3}$ is in consistency with the axis $\boldsymbol{y}_{2}$. As to the continuum manipulator, the local coordinate system $\boldsymbol{o}_{c}-\boldsymbol{x}_{c} \boldsymbol{y}_{c} \boldsymbol{z}_{c}$ is attached to the interface between the rigid shaft and the flexible continuum manipulator part (see Fig. 4). It is a moving coordinate system translating along the axis $z_{3}$ and the offset position of its origin 
$\boldsymbol{o}_{c}$ coincides with the origin $\boldsymbol{o}_{3}$, i.e. the center of the spherical mechanism. For simplicity, the axes $\boldsymbol{y}_{c}$ and $\boldsymbol{y}_{3}$ are overlapping in the case and the axis $z_{c}$ is collinear with the axis $z_{3}$ but in opposite directions.

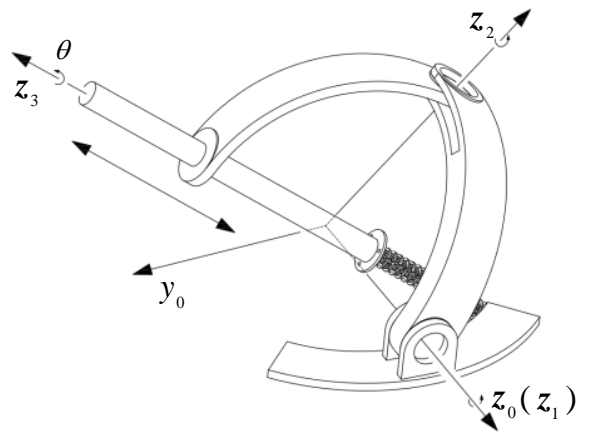

Figure 3. The simplified sketch of the proposed RCM mechanism attached with coordinate systems.

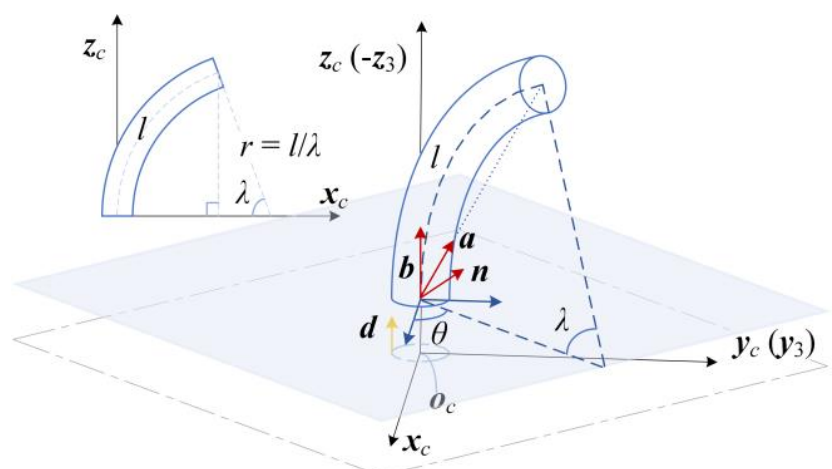

Figure 4. The sketch of the spatial bending configuration with the local coordinate system $\boldsymbol{o}_{c}-\boldsymbol{x}_{c} \boldsymbol{y}_{c} \boldsymbol{z}_{c}$.

Here, in the study of kinematics of the proposed robot, we consider two kinds of spaces - the operational space of end-effector and the configuration space of the mechanism, and the kinematic modeling is a static geometrical problem of computing the mutual relation between them. The end-effector is supposed to be mounted to the tip of the flexible continuum manipulator, thus the position and orientation will be considered with respect to the continuum manipulator tip for a general study. To avoid a complexed calculation, we do not consider the active contraction of the continuum manipulator in the following analysis.

\section{B. Coordinate System Transformation}

The motion of this RCM mechanism is determined by controlling four parameters, which are defined as $\varphi_{1}, \varphi_{2}, \theta$ and $d . \varphi_{1}$ and $\varphi_{2}$ are the two rotations about the axes $z_{1}$ and $z_{2}$; $\theta$ denotes the spinning angle of the shaft and $d$ represents its translation. The angle $\alpha_{1}$ between the axes $z_{1}$ and $z_{2}$ and the angle $\alpha_{2}$ between the axes $z_{2}$ and $z_{3}$ are both constant values and depend on the linkage design parameters of this spherical linkage RCM mechanism.

Suppose a vector $\boldsymbol{e}$ in local coordinate system $\boldsymbol{o}_{3}-\boldsymbol{x}_{3} \boldsymbol{y}_{3} z_{3}$ to be transformed to global coordinate system $\boldsymbol{o}_{o}-\boldsymbol{x}_{o} \boldsymbol{y}_{o} \boldsymbol{z}_{o}$. The following product of the rotation matrices in series is derived to achieve such operation [20]:

$$
\mathbf{R}=\mathbf{R}\left(z_{1}, \varphi_{1}\right) \cdot \mathbf{R}\left(\boldsymbol{y}_{1}, \alpha_{1}\right) \cdot \mathbf{R}\left(z_{2}, \varphi_{2}\right) \cdot \mathbf{R}\left(\boldsymbol{y}_{2}, \alpha_{2}\right)
$$

where $\mathbf{R}\left(z_{1}, \varphi_{1}\right)$ denotes the 3-by-3 rotation matrix to perform a rotation $\varphi_{1}$ about the axis $z_{1}$, in three dimensions, using the right hand rule. Other rotation matrices are represented in the same way in Eq. (1). The columns of the obtained rotation matrix $\mathbf{R}$ are the unit vectors of the local coordinate system $\boldsymbol{o}_{3}-\boldsymbol{x}_{3} \boldsymbol{y}_{3} \boldsymbol{z}_{3}$ written in global coordinate system $\boldsymbol{o}_{o}-\boldsymbol{x}_{o} \boldsymbol{y}_{o} \boldsymbol{z}_{o}$.

Thus, the description of a vector $\boldsymbol{e}$ with respect to the global coordinate system $\boldsymbol{o}_{o}-\boldsymbol{x}_{o} \boldsymbol{y}_{o} \boldsymbol{z}_{o}$ can be derived by R.e Besides, the transformation from the local coordinate system $\boldsymbol{o}_{c}-\boldsymbol{x}_{c} \boldsymbol{y}_{c} \boldsymbol{z}_{c}$ to the local coordinate system $\boldsymbol{o}_{3}-\boldsymbol{x}_{3} \boldsymbol{y}_{3} \boldsymbol{z}_{3}$ is a simple rotational operator $\mathbf{R}\left(\boldsymbol{y}_{c}, \pi\right)$.

In order to find the map that relates the continuum manipulator's configuration to its tip position and orientation, we firstly describe this relation in its local coordinate system $\boldsymbol{o}_{c}-\boldsymbol{x}_{c} \boldsymbol{y}_{c} \boldsymbol{z}_{c}$.

The configuration space variable of the continuum manipulator is defined as $\left[\begin{array}{lll}k & \theta & d\end{array}\right]^{\mathrm{T}}$, where $k$ is the bending curvature; $\theta$ is the shaft's spinning angle; $d$ denotes the shaft's translational displacement along spindle axis. In Fig. 4, the planar model of the continuum manipulator is illustrated. Given radius $r=1 / k$ and bending angle $\lambda=k \cdot l$, we thus have $x=(1-\cos (k \cdot l)) / k$ and $z=\sin (k \cdot l) / k$ in the $x_{c} z_{c}$ plane based on the trigonometry. After that, we rotate the planar model about the axis $\boldsymbol{z}_{c}$ and obtain a spatial model as shown in Fig. 4. The derivation of spatial position coordinates of the continuum manipulator in the local coordinate system $\boldsymbol{o}_{c}-\boldsymbol{x}_{c} \boldsymbol{y}_{c} \boldsymbol{z}_{c}$ is derived as follows

$$
\boldsymbol{x}_{c}=\left[\begin{array}{c}
x_{c} \\
y_{c} \\
z_{c}
\end{array}\right]=\mathbf{R}\left(z_{c}, \theta\right) \cdot\left[\begin{array}{c}
\frac{1-\cos k l}{k} \\
0 \\
\frac{\sin k l}{k}
\end{array}\right]=\left[\begin{array}{c}
\frac{\cos \theta(1-\cos k l)}{k} \\
\frac{\sin \theta(1-\cos k l)}{k} \\
\frac{\sin k l}{k}+d
\end{array}\right]
$$

Then, we can derive this tip position in global coordinate system $\boldsymbol{o}_{o}-\boldsymbol{x}_{o} \boldsymbol{y}_{o} \boldsymbol{z}_{o}$ via the following calculation

$$
\boldsymbol{x}=\mathbf{R} \cdot \mathbf{R}\left(\boldsymbol{y}_{c}, \pi\right) \cdot \boldsymbol{x}_{c}
$$

In order to describe the tip orientation of the continuum manipulator, we first define two unit vectors: $\boldsymbol{a}$ is along the position vector $\left[\begin{array}{lll}x_{c} & y_{c} & \left(z_{c}-d\right)\end{array}\right]^{\mathrm{T}}$ and $\boldsymbol{b}$ is along the axis $\boldsymbol{z}_{c}$. Taking the cross product $\boldsymbol{n}=\boldsymbol{b} \times \boldsymbol{a}$ and $|\boldsymbol{n}|=|\boldsymbol{b}| \cdot|\boldsymbol{a}| \cdot \sin \delta$, we find their normal vector $\boldsymbol{n}$ and also have the angle $\delta$ between the vector $\boldsymbol{b}$ and the vector $\boldsymbol{a}$. Rotate the unit vector $\boldsymbol{a}$ via Rodrigues' rotation formula [20]

$$
\mathbf{R}_{r}=\cos \delta \cdot \mathbf{I}+\sin \delta \cdot[\boldsymbol{n} \times]+(1-\cos \delta) \cdot \boldsymbol{n} \cdot \boldsymbol{n}^{\mathrm{T}}
$$

where I is a $3 \times 3$ identity matrix; $[\boldsymbol{n} \times]$ represents a skew-symmetric matrix spanned by the normal vector $\boldsymbol{n}$. 
Thus, the description of the tip orientation with respect to global coordinate system $\boldsymbol{o}_{o}-\boldsymbol{x}_{o} \boldsymbol{y}_{o} \boldsymbol{z}_{o}$ can be formulated via a direction vector

$$
\gamma=\mathbf{R} \cdot \mathbf{R}\left(\boldsymbol{y}_{c}, \pi\right) \cdot \mathbf{R}_{r} \cdot \boldsymbol{a}
$$

So far, both the position (Eq. (3)) and orientation (Eq. (5)) of the effector attached to the entire manipulator system in global coordinate system have been analytically formulated.

\section{ANALYSIS}

\section{A. Workspace Analysis}

In most conventional cases, the rigid instrument's orientations are only achieved by rotating the hinged joints of the spherical linkage RCM mechanism, and the tip orientation cannot be adjusted locally. Here, with the compact combination of a continuum manipulator and an RCM mechanism, the workspace of the entire system not only becomes enlarged to a great extent, but also produces a "dexterous operation area", where the manipulator system has redundant motion DOFs, i.e. each tip position in this area can be realized with more than one configurations.

Figure 5 illustrates the tip workspace of the proposed manipulator system, which is simulated in MATLAB based on Eq. (3). The design parameters and control variables are assigned with the values that are listed in Tab. I. The total workspace volume is estimated by calculating its fitting cuboid as follows

$$
V_{\text {reachable }}=160 \mathrm{~mm} \times 160 \mathrm{~mm} \times 120 \mathrm{~mm} \times \eta=2.15 \times 10^{6} \mathrm{~mm}^{3}
$$

where $\eta$ is the correction factor and in this case it is empirically set to be 0.7 .

Similarly, the volume of the "dexterous operation area" (fitted by the smaller cuboid in Fig. 5) is also estimated, which equals $1.73 \times 10^{6} \mathrm{~mm}^{3}$ when the correction factor takes the value of 1.2. It can be seen that the "dexterous operation area" makes up $80 \%$ of the total reachable workspace. Thus, the added kinematic redundancy allows the robotic system in almost the whole workspace to enhance the dexterity inside the patient's body - a desirable feature in MIS and potentially improving the safety.

For further comparisons, Fig. 6 and Fig. 7 respectively illustrate the workspace of a separate continuum manipulator with restricted translational displacements and a traditional spherical linkage RCM mechanism. The workspace in Fig. 6 is plotted in MALTAB according to Eq. (2); the workspace in Fig. 7 is plotted according to the formula $\boldsymbol{x}=\mathbf{R} \cdot \mathbf{R}\left(\boldsymbol{y}_{c}, \pi\right) \cdot \boldsymbol{d}$, which is similar to Eq. (3).

TABLE I. TABLE TyPe Styles The Assigned VAlue/RAnge OF PARAMETERS/INPUT VARIABLES.

\begin{tabular}{cc|cc}
\hline \hline Const. parameters & Value & Variables & Range \\
\hline$\alpha_{1}$ & $\pi / 2$ & $\varphi_{1}$ & {$[-\pi / 4, \pi / 4]$} \\
$\alpha_{2}$ & $\pi / 4$ & $\varphi_{2}$ & {$[-4 \pi / 5,4 \pi / 5]$} \\
$l$ & $50 \mathrm{~mm}$ & $\theta$ & {$[0,2 \pi]$} \\
radius of sphere & $100 \mathrm{~mm}$ & $\lambda(=k \cdot l)$ & {$[0, \pi]$} \\
& & $d$ & {$[0,50 \mathrm{~mm}]$} \\
\hline \hline
\end{tabular}

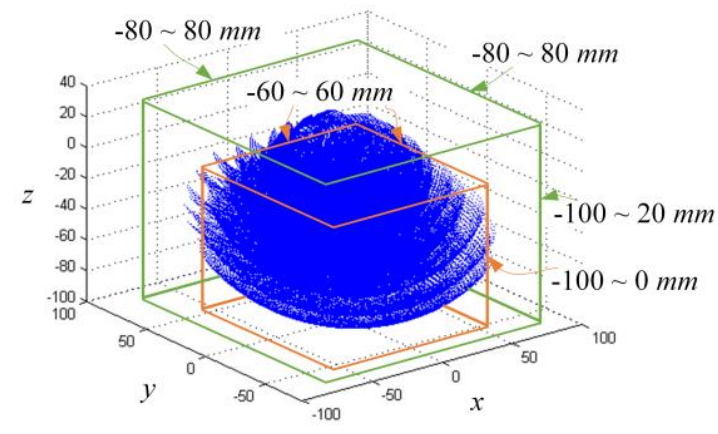

Figure 5. The tip workspace (blue-color-dotted space) of the proposed manipulator system which integrates the continuum manipulator with the spherical linkage RCM mechanism. The volume of the reachable workspace is estimated by fitting it into a cuboid space. (Unit: $\mathrm{mm}$ )

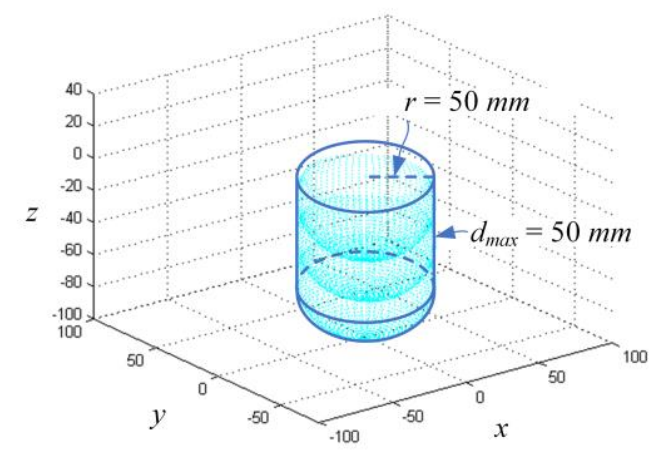

Figure 6. The tip workspace (cyan-color-dotted space) of the continuum manipulator only. The translated displacement is up to $50 \mathrm{~mm}\left(d_{\max }\right)$. The area is fitted by a cylinder (radius is denoted by $r$ ) and a hemisphere. (Unit: $\mathrm{mm}$ )

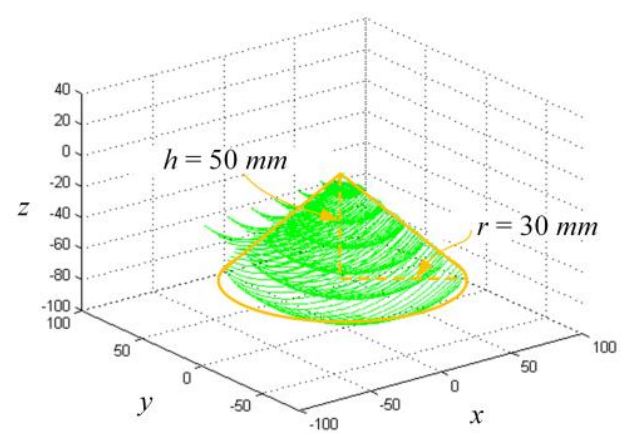

Figure 7. The tip workspace (lime-color-dotted space) of the spherical linkage RCM mechanism with only a rigid shaft and the maximum penetration length is determined as $100 \mathrm{~mm}$ (that is different with other cases in our analysis). Its reachable workspace is fitted by a cone. The lateral height of the fitted cone space is denoted by $h$ and the radius of the cone is denoted by $r$. (Unit: $\mathrm{mm}$ )
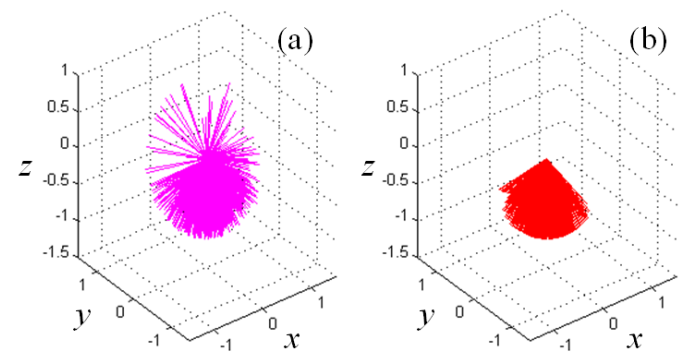

Figure 8. Illustrating the controllable 3D orientations with (a) the proposed robot at the tip position $[-135,50,50]$ and (b) spherical RCM mechanism only. (Unit: $\mathrm{mm}$ ) 
Comparing all the three workspaces depicted in Fig. 5, Fig. 6, and Fig. 7, it is obvious that the proposed manipulator system significantly increases the reachable workspace of its end-effector. The volume of the continuum manipulator's workspace in Fig. 6 is calculated as $6.54 \times 10^{5} \mathrm{~mm}^{3}$ under the same design parameter and corresponding control input. This calculated result just takes about $37 \%$ of the previously-identified "dexterous operation area" of its integrated RCM mechanism. Thus, mounting to the spherical linkage RCM mechanism equivalently enables the continuum manipulator with more direction selections to translate its bending configurations. Due to the actual workspace volume for of the spherical linkage RCM mechanism with only a rigid shaft depends on the length of the attached instrument, thus it varies case by case and we do not make a numerical comparison here.

Figure 8(a) shows all the possible orientations of the instrument tip controlled by the proposed robot at one example tip point. Given any positions within the reachable workspace, we can theoretically calculate all the possible tip orientations based on the following derivations building upon Eqs. (1)-(5). These orientations are described in the local coordinate system $\boldsymbol{o}_{t}-\boldsymbol{x}_{t} \boldsymbol{y}_{t} \boldsymbol{z}_{t}$ of the tip as seen in Fig. 9.

First, the given position in the global coordinate system is presented by $\boldsymbol{x}=[x, y, z]^{\mathrm{T}}$ and its forward derivation is given by Eq. (3). Then, we derive the coordinates of the tip location in Fig. 9; the following transformations apply.

a) The coordinates in the bending-only configuration are

$$
\boldsymbol{x}_{c}^{\prime}=\mathbf{R}\left(\boldsymbol{y}_{c}, \pi\right)^{-1} \cdot \mathbf{R}^{-1} \cdot \boldsymbol{x}-\boldsymbol{d}
$$

where $\boldsymbol{d}=\left[\begin{array}{lll}0 & 0 & d\end{array}\right]^{\mathrm{T}}$; this step eliminates the effect of translational displacements.

b) The coordinates in $2 \mathrm{D}$ bending plane (exactly what we see in Fig. 9) are

$$
\boldsymbol{x}_{c}^{\prime \prime}=\mathbf{R}\left(z_{c}, \theta\right)^{-1} \cdot \boldsymbol{x}_{c}^{\prime}
$$

Suppose that $\boldsymbol{x}_{c}^{\prime \prime}=\left[\begin{array}{lll}x_{c}^{\prime \prime} & 0 & z_{c}^{\prime \prime}\end{array}\right]^{\mathrm{T}}$ which is described by $\boldsymbol{x}$ $=[x, y, z]^{\mathrm{T}}$, then

$$
r=\frac{x_{c}^{\prime \prime 2}+z_{c}^{\prime \prime 2}}{2 x_{c}^{\prime \prime}}
$$

The last step is to describe the tip orientation in its local coordinate system. We suppose there is a unit vector $\boldsymbol{s}_{t}$ as seen in Fig. 9. By use of similar triangle calculations, we can have

$$
\boldsymbol{s}_{t}=\left[\begin{array}{lll}
\frac{z_{c}^{\prime \prime}}{r} & 0 & \frac{r-x_{c}^{\prime \prime}}{r}
\end{array}\right]^{\mathrm{T}}
$$

Then, the tip orientations of this continuum manipulator system are formulated by

$$
s=\mathbf{R} \cdot \mathbf{R}\left(z_{c}, \theta\right) \cdot s_{t}
$$

Figure $8(\mathrm{~b})$ is given for comparisons, which illustrates all the orientations restrained at the origin. This is considered to be one drawback of the single RCM mechanism. In contrast, the proposed robot system enables the manipulator tip locally adjusting its orientation in almost parts of its workspace. According to Eq. (10), given any position $\boldsymbol{x}=[x, y, z]^{\mathrm{T}}$ within the workspace, all the possible orientations can be calculated.

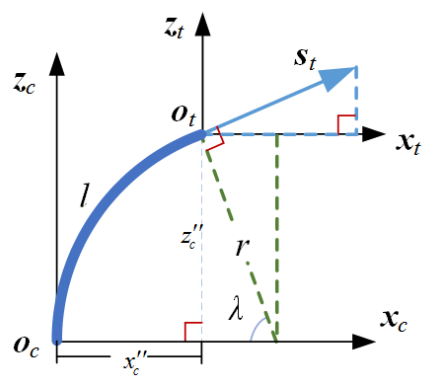

Figure 9. Schematic diagram of the bending configuration of a continuum manipulator ( $l$ in length and $\lambda$ in bending angle) in 2D plane.

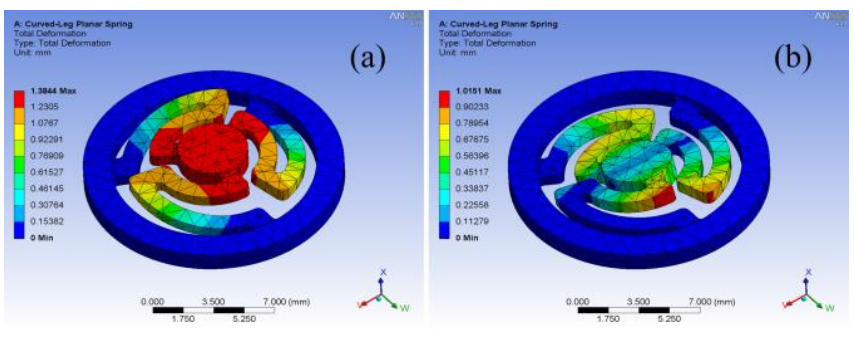

Figure 10. FEA simulations: (a) translational and (b) rotational deflections when force and moment respectively apply.

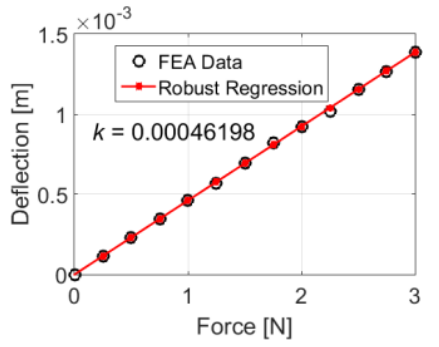

(a)

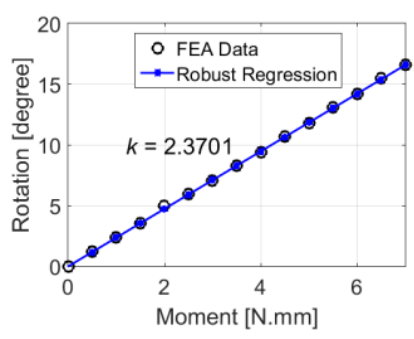

(b)
Figure 11. Numerical results of FEA simulations. The robust regression technique is used to predict (a) translational and (b) rotational compliance.

\section{B. Finite Element Analysis (FEA)}

The curved-leg planar spring is for the first time introduced for continuum manipulator design in this paper. In order to study its compliance characteristics, here we employ the FEA technique. The FEA simulation of the planar spring is conducted using the commercial software package ANSYS ${ }^{\circledR}$. Two types of loads are applied to the central platform and the ring base is fixed, thus generating the rotational and translational deflections (see Fig. 10). All the important features of deformation of the part are obtained in static structural analysis environment.

According to the simulation results, both translational and rotational compliance are revealed in Fig. 11, where we can see that the lateral translational compliance is equal to $4.6 \times 10^{-4} \mathrm{~m} / \mathrm{N}$ and the out-plane rotational compliance is equal to $2.37^{\circ}$ (N.mm). Those values are calculated via the robust regression technique. Besides, the plotted figures confirm that the deflections scale linearly with the applied loads.

\section{PREliminary TeSt AND Discussion}

In this section, we describe an overview of preliminary tests validating the design. The continuum manipulator 
skeleton and spherical linkages are fabricated using a rapid prototyping machine. The entire system is built as seen in Fig. 12. This continuum manipulator system with an RCM mechanism as a whole exhibited motion of the remote center around the center of the sphere. By separately actuating the continuum manipulator itself, the system further increases maneuverability. Owing to the redundant DOFs, more than one tip orientations can be achieved with this system when fixing the manipulator tip at a point in workspace. The functionality of this proposed robotic system is validated.

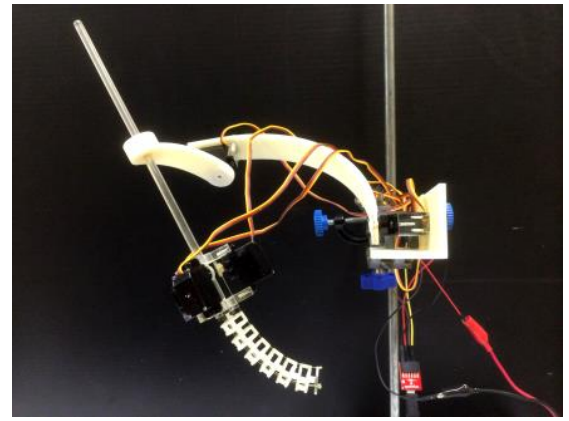

Figure 12. A 3D-printed prototype for preliminary tests. The two rotational DOFs of the spherical linkage mechanism are actuated with steering motors; the mounted continuum manipulator is articulated via there tendons.

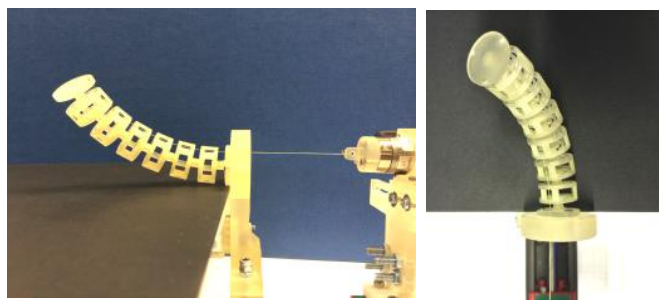

Figure 13. Continuum manipulator bending tests.

Figure 13 shows the bending tests of the continuum manipulator. The profile of the continuum manipulator backbone fits a constant curve, whilst remaining the same backbone length during bending. When the manipulator is activated for bending deflections, the compliance is exhibited, however, the stiffness seriously limited. This will become a problem when exacting a certain force inside the body, even combining the spherical linkages is of no help. Next, the continuum manipulator is actuated via three tendons, thus, it can be deflected in $3 \mathrm{D}$ space. We know that the spherical linkage mechanism has one rotational DOF about the rigid shaft. This DOF actually can be eliminated in order to reduce the mechanical actuation complicity, and it will also potentially simplify the control scheme.

\section{CONCLUSION AND FUTURE WORK}

This paper presents the conceptual design of a continuum manipulator system integrated an RCM mechanism. The kinematics of the entire system is derived. Based on workspace analysis, it reveals that this robot not only enlarges the reachable workspace but also increases the operational dexterity, which indicates the potential to benefit the MIS procedures. A 3D-printed prototype has been built for preliminary tests. Future work will be focused on further improving the system integration and optimizing the design according to the requirements of specific MIS cases and practical design specifications. Besides, a metallic physical prototype will be fabricated for further experiments.

\section{REFERENCES}

[1] J. S. Dai, "Surgical robotics and its development and progress," specia issue on surgical robotics, system development, application study and performance analysis, Robotica, vol. 28, no. 2, 2010.

[2] R. H. Taylor and D. Stoianovici, "Medical robotics in computer integrated surgery," IEEE Trans. Robot. Autom., vol. 19, no. 5, pp. 765-781, 2003.

[3] C. Shi, C. Tercero, S. Ikeda, K. Ooe, T. Fukuda, K. Komori, et al., "In vitro three-dimensional aortic vasculature modeling based on sensor fusion between intravascular ultrasound and magnetic tracker," Int. J. Med. Robot, vol. 8, no. 3, pp. 291-9, 2012.

[4] C. Shi, C. Tercero, X. Wu, S. Ikeda, K. Komori, K. Yamamoto, et al., "Real-time in vitro intravascular reconstruction and navigation for endovascular aortic stent grafting," Int. J. Med. Robot, DOI: 10.1002/rcs.1736, Feb 92016.

[5] J. Burgner-Kahrs, D. C. Rucker, and H. Choset, "Continuum robots for medical applications: A survey," IEEE Trans. Robot., vol. 31, no. 6, pp. 1261-1280, 2015

[6] R. J. Webster, R. Joseph, and N. J. Cowan, "Mechanics of precurved-tube continuum robots," IEEE Trans. Robot., vol. 25, no. 1, pp. 67-78, 2009.

[7] K. Xu and N. Simman, "An investigation of the intrinsic force sensing capabilities of continuum robots," IEEE Trans. Robot., vol. 24, no.3, pp. 576-587, 2008.

[8] I. D. Walker, "Continuous backbone "continuum" robot manipulators," ISRN Robotics, vol. 2013, Article ID 726506, 19 pages, 2013.

[9] C.-H. Kuo, J. S. Dai, and P. Dasgupta, "Kinematic design considerations for minimally invasive surgical robots: an overview," Int. J. Med. Robot., vol. 8, no. 2, pp. 127-145, 2012.

[10] Remote center-of-motion robot for surgery, by R. H. Taylor, J. Funda, D. Grossman, J. Karidis, and D. LaRose. (1995, Mar 14). U.S. Patent 5397323 [Online]. http://www.google.com/patents/US5397323

[11] M. J. H. Lum, J. Rosen, M. N. Sinanan, and B. Hannaford, "Optimization of a spherical mechanism for a minimally invasive surgical robot: theoretical and experimental approaches," IEEE Trans. Biomed. Eng., vol. 53, no. 7, pp. 1440-1445, 2006.

[12] H. Choi, H. J. Kim, Y.-A. Lim, H.-S. Kwak, J.-W. Jang, and J. Won, "Conically shaped remote center-of-motion mechanism for single-incision surgery," in Proc. IEEE/RSJ Int. Conf. Intelligent Robots and Systems, Tokyo, Japan, 2013, pp. 3604-3609.

[13] M. Piccigallo, U. Scarfogliero, C. Quaglia, G. Petroni, P. Valdastri, A Menciassi, and P. Dario, "Design of a novel bimanual robotic system for single-port laparoscopy,” IEEE/ASME Trans. Mechatronics, vol. 15, no. 6, pp. 871-878, 2010.

[14] P. Berkelman and J. Ma, "A compact modular teleoperated robotic system for laparoscopic surgery," Int. J. Robotics Res., vol. 28, no. 9, pp. 1198-1215, 2009.

[15] T. Yoshikawa, K. Hosoda, T. Doi, and H. Murakami, "Quasi-static trajectory tracking control of flexible manipulator by macro-micro manipulator system," in Proc. IEEE Int. Conf. Robotics and Automation, 1993, pp. 3012-3017.

[16] P. Qi, C. Qiu, H. Liu, J. S. Dai, L. Seneviratne, and K. Althoefer, "A novel continuum-style robot with multilayer compliant modules," in Proc. IEEE/RSJ Int. Conf. Intell. Robots Syst., 2014, pp. 3175-3180.

[17] J. J. Parise, L. L. Howell, and S. P. Magleby, "Ortho-planar Linear-motion Springs," Mechanism and machine theory, vol. 36, no. 11, pp. 1281-1299, 2001.

[18] J. Shi, P. Mederic, V. Pasui, G. Morel, and S. Wang, "Preliminary results on the design of a novel laparoscopic manipulator," in Proc. 11th World Congr. in Mech. and Mach. Sci., 2002.

[19] M. Lum, C. Friedman, G. Sankaranarayanan, H. King, K. Fodero, R. Leuschke, B. Hannaford, J. Rosen, and M. Sinanan, "The RAVEN: design and validation of a telesurgery system," Int. J. Robotics Res., vol. 28, no. 9, pp. 1183-1197, 2009.

[20] J. S. Dai, Geometrical Foundations and Screw Algebra for Mechanisms and Robotics, Chinese translations, Higher Education Press, Beijing, ISBN: 9787040334838, 2014. 